\title{
The Invariant Charges of the Nambu-Goto Theory: Their Geometric Origin and Their Completeness
}

\author{
K. Pohlmeyer ${ }^{1}$ and K.-H. Rehren ${ }^{2}$ \\ 1 Fakultät für Physik der Universität Freiburg, Hermann-Herder-Strasse 3, D-7800 Freiburg, \\ Federal Republic of Germany \\ ${ }^{2}$ Institut für Theorie der Elementarteilchen, Fachbereich Physik, Freie Universität Berlin, \\ Arnimallee 14, D-1000 Berlin 33, Federal Republic of Germany
}

\begin{abstract}
We give an alternative construction of the reparametrization invariant "non-local" conserved charges of the Nambu-Goto theory which elucidates their geometric nature and their completeness property.
\end{abstract}

\section{Introduction}

In a series of publications [1-3] the present authors have shown that the classical Nambu-Goto string theory possesses infinitely many independent, reparametrization invariant, "non-local" conserved charges $\mathscr{Z}_{\mu_{1} \ldots \mu_{N}}^{ \pm(K)}$, which serve as infinitesimal generators of active symmetry transformations. The Poisson algebra of these "invariant charges" $\mathscr{Z}_{\mu_{1} \ldots \mu_{N}}^{ \pm(K)}$ has been analyzed in great detail $[2,4]$.

In the present article we shall give a geometric interpretation of the invariant charges of the Nambu-Goto string in its Euclidean version. In particular, we shall address the question to what extent a given choice of values for the invariant charges fixes the "trajectory" surface of the string in $d$-dimensional Euclidean space $\mathbb{R}^{d}$.

The geometric interpretation will be formulated in terms of homology and cohomology classes: A given complete minimal ("trajectory") surface $\Sigma$ can be complexified to a Riemann surface in $\mathbb{C}^{d}$. This surface can be described by a $d$-tuple of multi-valued analytic functions $w_{\mu}=w_{\mu}(z)$ such that $w_{\mu}^{\prime}(z)=\frac{d}{d z} w_{\mu}(z)$ are singlevalued analytic functions in a canonical region $\Omega$ satisfying the complex constraint equation $\sum_{\mu} w_{\mu}^{\prime 2}(z)=0$ for $z \in \Omega[5]$. The summation extends from $\mu=1$ to $\mu=d$. The canonical region $\Omega$ typically has the form of an annulus $1<|z|<e^{\lambda_{1}}$ minus $(n-2)$ concentric circular (two-sided) slits along a connected part of: $|z|=e^{\lambda_{2}}$, $i=2, \ldots, n-1 ; \lambda_{1}>\lambda_{2} \geqq \lambda_{3} \geqq \ldots \geqq \lambda_{n-1}>0 ; n=2,3, \ldots, \infty[6,7]$. The values of the invariant charges for the given minimal surface are periods of certain analytic differentials - constructed from the $d$-tuple of analytic functions - along a closed curve $\gamma$ in the canonical region (corresponding to a closed curve $\mathscr{C}$ on the minimal surface $\Sigma$ in $\mathbb{R}^{d}$ ). As such they depend on the curve $\gamma$ only via its homology class $[\gamma]$. This is the Euclidean version of the statement that in Minkowski space the charges in question are reparametrization invariant and conserved. 
In general, to one and the same minimal surface several different value sets for the collection of the invariant charges are assigned depending on the choice of the homology class $[\gamma]$. In Minkowski space the necessity to distinguish different homology classes reflects the fact that the trajectory surface may branch and merge (except trajectories which are the Minkowski counterparts of catenoids). What locally looks like a one string state may globally be just a part of a scattering configuration.

We have considered two important types of situations. In the first instance we have considered the class $\mathscr{I}$ of (complete) minimal surfaces $\Sigma$ for which it is possible to find a branch $\Sigma^{\prime} C \Sigma$ of the type of an annulus and a family of parallel hyperplanes $E_{h}=\left\{x \in \mathbb{R}^{d} \mid x \cdot e=h\right\}$ for some unit vector $e$ and some interval $h_{1}<h<h_{2}$ such that all curves $\mathscr{C}_{h}$ of intersection of $\Sigma^{\prime}$ with $E_{h}$ are Jordan curves [5]. The curve $\gamma$ was chosen from the homology class of the "trace" $\gamma_{h}$ of $\mathscr{C}_{h}$ in $\Omega$. In this case we were able to show that the complete set of values of the invariant charges determines the (complete) minimal surface uniquely up to global translations in $\mathbb{R}^{d}$.

In this context let us remind the reader of the situation in classical mechanics: An (autonomous) integrable Hamiltonian system with a finite number $f$ of degrees of freedom possesses at most $(2 f-1)$ independent constants of motion. By fixing their values within the allowed range, the trajectory curve of the system is uniquely determined. Only $f$ independent constants of motion are in involution. Their values determine the invariant tori. Thus the totality of invariant charges of the Nambu-Goto theory corresponds to the above set of $(2 f-1)$ independent constants of motion (taking into account the fact that the invariant charges do not involve the absolute position of the string). It is the maximal abelian subalgebra of invariant charges identified in [4] which determines the invariant tori for a string.

In the second instance we have considered the following situation: A smooth Jordan curve $\mathscr{C}$ forms the boundary of a simply connected part $\Sigma^{\prime}$ (of the type of a disc) of a minimal surface $\Sigma$. Let $\gamma$ be the smooth Jordan curve in parameter space corresponding to $\mathscr{C}$. In this case all invariant charges vanish, and certainly with their help one cannot distinguish among different (complete) minimal surfaces. Under some assumptions which we believe to be purely technical we can show that also the converse holds: The vanishing of all invariant charges implies that $\mathscr{C}$ effectively does not wrap around (a branch of) the minimal surface $\Sigma$ but rather resides in a simply connected region of $\Sigma$.

General Euclidean situations will be neither of the first nor of the second type. However, the first type of situation is of considerable interest in view of the original Minkowski version of the Nambu-Goto string with the initial data given on some plane $t=x^{0}=$ const (or rather on some space-like surface) and the energymomentum density restricted to the forward light-cone. The second type of situation has a bearing on the symmetry and uniqueness of the ground state of the string theory [3].

\section{The Construction and Interpretation of the Invariant Charges}

Our first goal is the construction of the analytic differentials mentioned in the introduction. For that we start from the $d$-tuple of multi-valued analytic functions

$$
w_{\mu}=w_{\mu}(z)=\varphi_{\mu}(z)+i \psi_{\mu}(z), \quad z \in \Omega,
$$


describing the Riemann surface in $\mathbb{C}^{d}$. To make contact with the data of the Nambu-Goto theory, in particular with the formalism of [1-4], choose a (regular) point $y$ on $\Sigma$ and a unit tangent vector of $\Sigma$ passing through it. Then draw a smooth curve $\mathscr{C}$ passing through the given point in the given direction. In parameter space this corresponds to the choice of a point $\zeta \in \Omega$, a direction in the complex plane and a smooth curve $\gamma \subset \Omega: z=z(\sigma)$ passing through $\zeta$ in the chosen direction when $\sigma$ takes the value zero.

$$
\mathscr{C}: \quad x_{\mu}=\varphi_{\mu}(z(\sigma)), \quad y_{\mu}=\varphi_{\mu}(z(0)) .
$$

By covering a neighbourhood $U$ of the point $y$ on the minimal surface $\Sigma$ by curves $\mathscr{C}_{\tau}$ which do not intersect themselves nor their alikes $\left(\mathscr{C}=\mathscr{C}_{\tau=0}\right)$ and which correspond to curves $\gamma_{\tau} \subset \Omega: z=z(\tau, \sigma)$ with similar properties $\left(\gamma=\gamma_{\tau=0}\right)$ and a positive orientation of the zweibein field $\partial_{\tau} z, \partial_{\sigma} z$, the differentials $d w_{\mu}$ along an infinitesimal arc of the curve $\gamma$ near $\zeta$ are related to the string degrees of freedom as follows

$$
d w_{\mu}=\left.\frac{d w_{\mu}(z)}{d z}\right|_{z=z(\tau, \sigma)} d z(\tau, \sigma)=\left\{\frac{\partial}{\partial \sigma} x_{\mu}(\tau, \sigma)+i p_{\mu}(\tau, \sigma)\right\} d \sigma
$$

with $\tau=\sigma=0$. (The string tension parameter $M^{2}$ has been set equal to 1.)

Next, we define complex functions $W_{\mu_{1} \ldots \mu_{N}}(\zeta)$ on $\Omega$ by fixing a homology class $\left[\gamma_{0}\right]$ and choosing for each $\zeta \in \Omega$ an oriented closed parametrized curve $(\subset \Omega)$ through $\zeta: \gamma_{\zeta} \in\left[\gamma_{0}\right]$. Let the parametrization be given by some periodic function $z=z_{\zeta}(\sigma), 0 \leqq \sigma \leqq 2 \pi$, such that

$$
z_{\zeta}(0)=z_{\zeta}(2 \pi)=\zeta
$$

Let

$$
W_{\mu_{1} \ldots \mu_{N}}(\zeta)=W_{\mu_{1} \ldots \mu_{N}}^{\gamma_{\zeta}}(\zeta) \doteq \int_{0}^{2 \pi} d \sigma_{1} \int_{0}^{\sigma_{1}} d \sigma_{2} \ldots \int_{0}^{\sigma_{N}-1} d \sigma_{N} \prod_{i=1}^{N}\left[\left.w_{\mu_{l}}^{\prime}\left(z_{\zeta}(\sigma)\right) \frac{d z_{\zeta}(\sigma)}{d \sigma}\right|_{\sigma=\sigma_{l}}\right]
$$

It is rather easy to verify that $W_{\mu_{1} \ldots \mu_{N}}(\zeta)$ is independent of

i) the specific parametrization;

ii) the specific choice of a curve $\gamma_{\zeta}$ from $\left[\gamma_{0}\right]$ passing through $\zeta: W_{\mu_{1} \ldots \mu_{N}}^{\gamma_{\zeta}}(\zeta)$ $=W_{\mu_{1} \ldots \mu_{N}}^{\left[\gamma_{0}\right]}(\zeta)$,

and that $W_{\mu_{1} \ldots \mu_{N}}^{\left[\gamma_{0}\right]}(\zeta)$ is a single-valued analytic function on $\Omega$. Actually, the function $W_{\mu_{1} \ldots \mu_{N}}^{\left[\gamma_{0}\right]}(\zeta)$ is identical with the tensor component $\mathscr{R}_{\mu_{1} \ldots \mu_{N}}(\tau, 0)$ of [1-4]. Hence we know from our previous analysis [2] that

$$
d W_{\mu_{1} \ldots \mu_{N}}^{\left[\gamma_{0}\right]}(z)=d w_{\mu_{1}}(z) W_{\mu_{2} \ldots \mu_{N}}^{\left[\gamma_{0}\right]}(z)-W_{\mu_{1} \ldots \mu_{N-1}}^{\left[\gamma_{0}\right]}(z) d w_{\mu_{N}}(z) .
$$

This equation implies that a cyclic permutation of the tensor indices changes the analytic differential $d w_{\mu_{1}}(z) W_{\mu_{2} \ldots \mu_{N}}^{\left[\gamma_{0}\right]}(z)$ by a total differential. In other words, a cohomology class contains along with $d w_{\mu_{1}}(z) W_{\mu_{2} \ldots \mu_{N}}^{\left[\gamma_{0}\right]}(z)$ all those analytic differentials which can be obtained from the latter by cyclic permutations of the tensor indices. The invariant charges $\mathscr{Z}_{\mu_{1} \ldots \mu_{N}}$ are just the periods

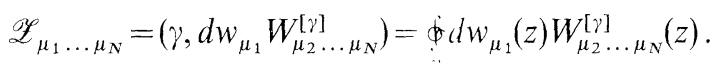

As such they depend on the cycles $\gamma$ and cocycles $d w_{\mu_{1}} W_{\mu_{2} \ldots \mu_{N}}^{[\gamma]}$ only via the corresponding homology class $[\gamma]$ and cohomology class $\left[d w_{\mu_{1}} W_{\mu_{2} \ldots \mu_{N}}^{[\gamma]}\right]$, respectively. In particular, they codify information about the topological properties of 
the Riemann surface in $\mathbb{C}^{d}$ and thereby [5] of the minimal surface $\Sigma$ in $\mathbb{R}^{d}$. A charge different from zero signals a non-trivial topology.

Apart from some elementary cases, e.g.

$$
\mathscr{Z}_{\mu}=\left(\gamma, d w_{\mu}\right)=i \mathscr{P}_{\mu},
$$

where the symbol $\mathscr{P}_{\mu}$ stands for the "energy-momentum" vector, the invariant charges generate symmetry transformations in "phase space" and not just in configuration space.

\section{Minimal Surfaces of Class $\mathscr{I}$ and Their Invariant Charges}

In this section we shall study the problem to what extent the invariant charges, evaluated along some fixed curve $\gamma$ of non-trivial homology, determine the minimal surface $\Sigma$. By an explicit construction we shall establish the following result: Given a complete set of values for the invariant charges $\mathscr{Z}_{\mu_{1} \ldots \mu_{N}}$, $\mu_{i}=1, \ldots, d, i=1, \ldots, N, N=1,2, \ldots$, compatible with the periods of the analytic differentials $d w_{\mu_{1}} W_{\mu_{2} \ldots \mu_{N}}^{\left[\gamma_{h}\right]}$ for some (complete) minimal surface $\Sigma$ of class $\mathscr{I}$ evaluated along the "trace" $\gamma_{h}$ of the Jordan curve $\mathscr{C}_{h}$ of intersection of an appropriate branch $\Sigma^{\prime}$ of $\Sigma$ with some hyperplane $E_{h}$. Then the given values for the invariant charges determine the minimal surface $\Sigma$ uniquely up to global translations in $\mathbb{R}^{d}$, and thus provide maximal observable information.

Proof. For simplicity we shall assume that the hyperplanes $E_{h}$ are perpendicular to the $d$-axis of a Cartesian coordinate system in $\mathbb{R}^{d}$ and that $h_{2}=-h_{1}=\frac{H}{2}>0$. This can certainly be arranged by an appropriate choice of the coordinate system in $\mathbb{R}^{d}$. According to [5] there exist "isothermal" parameters $\zeta=e^{\tau+i \sigma}$ which parametrize the subsurface $\Sigma_{H}$ of $\Sigma^{\prime} \subset \Sigma$ :

on an annulus

$$
\Sigma_{H} \doteq \bigcup_{-H / 2} \mathscr{C}_{h}
$$

$$
A=\left\{\zeta \in \mathbb{C}\left|e^{-\mu / 2}<\right| \zeta \mid<e^{+\mu / 2}\right\}
$$

with some modulus $\mu>0$ such that

$$
x_{d}(\tau, \sigma)=\Phi_{d}(\zeta)=\varphi_{d}(z(\zeta))=(H / \mu) \cdot \tau .
$$

Here $z(\zeta)$ is a holomorphic function for $\zeta \in A$ mapping $A$ on a two-fold connected subregion of $\Omega$. The isothermal property of the parameters $\tau$ and $\sigma$ implies

$$
\begin{gathered}
p_{d}(\tau, \sigma)=\partial_{\tau} x_{d}(\tau, \sigma)=H / \mu, \\
d w_{d}(\zeta)=(H / \mu) \cdot d(\tau+i \sigma) .
\end{gathered}
$$

In $A$ we expand $d w_{\mu}(z(\zeta))$ in a Laurent series,

$$
d w_{\mu}=\sum_{n=-\infty}^{+\infty} a_{\mu}^{n} \zeta^{n} d \zeta=\sum_{n=-\infty}^{+\infty} \alpha_{\mu}^{n} e^{n(\tau+i \sigma)} d(\tau+i \sigma) / i
$$

where $\alpha_{\mu}^{n}=i a_{\mu}^{n-1}$ and $\alpha_{d}^{n}=\delta_{n, 0} i(H / \mu)$. 
Next, we shall compute the invariant charges along the circle $\tau=0$ in terms of the Fourier coefficients $\alpha_{\mu}^{n}$. Due to the invariance of the charges under the reparametrization $\zeta \rightarrow \lambda \cdot \zeta, \lambda \in \mathbb{C} \backslash\{0\}$, the result is left unchanged if we replace the set $\alpha_{\mu}^{n}$ by the set $\lambda^{-n} \alpha_{\mu}^{n}$. Apart from this ambiguity, all coefficients $\alpha_{\mu}^{n}$ are determined by the complete set of non-trivial values for the invariant charges.

First note:

$$
\begin{gathered}
i \mathscr{P}_{d}=\oint d w_{d}=2 \pi \alpha_{d}^{0}=2 \pi i(H / \mu) \neq 0, \\
i \mathscr{P}_{j}=\oint d w_{j}=2 \pi \alpha_{j}^{0}, \quad j=1, \ldots, d-1 .
\end{gathered}
$$

Next, compute $(j, k=1, \ldots, d-1 ; N \geqq 2)$ :

$$
\begin{aligned}
\mathscr{Z}_{\underbrace{}_{N-2} \ldots d j k} & =\left(\alpha_{d}^{0}\right)^{N-2} \oint d \sigma_{1} \int_{\sigma_{1}}^{\sigma_{1}+2 \pi} d \sigma_{2} \int_{\sigma_{1}}^{\sigma_{2}} d \sigma_{3} \ldots \int_{\sigma_{1}}^{\sigma_{N-1}} d \sigma_{N} \sum_{m} \alpha_{j}^{m} e^{i m \sigma_{N-1}} \sum_{n} \alpha_{k}^{n} e^{i n \sigma_{N}} \\
& =\left(i \frac{H}{\mu}\right)^{N-2}\left\{\frac{(2 \pi)^{N}}{(N-1) !} \alpha_{j}^{0} \alpha_{k}^{0}-\sum_{m}^{\prime} \sum_{n}^{\prime} \delta_{m+n, 0} \alpha_{j}^{m} \alpha_{k}^{n} \sum_{K=2}^{N-1} \frac{(2 \pi)^{K}}{(K-1) !}\left(\frac{1}{i m}\right)^{N-K}\right\},
\end{aligned}
$$

where the symbol $\sum_{m}^{\prime}$ stands for summation over all integer values of $m$ from $-\infty$ to $+\infty$ with the exception of zero.

The term proportional to $(2 \pi)^{K}$ corresponds exactly to the charge $\mathscr{Z}_{d \ldots d j k}^{(K)}[2]$. This can be seen by considering the effect of integrating $p$ times around the circle $\tau=0$. The effect is, on the one hand, a simple replacement of $(2 \pi)$ by $p \cdot(2 \pi)$ in the above formula and, on the other hand, a replacement of the logarithm of the Lax monodromy matrix $\ln \Phi[2]$ by $\ln \Phi^{p}=p \cdot \ln \Phi$. Finally, recall that $\mathscr{Z}^{(K)}$ is generated by $\operatorname{tr}(\ln \Phi)^{K}$. We conclude

$$
\underbrace{\mathscr{Z}_{d \cdots d j k}^{(2)}}_{N-2}=-\left(\frac{H}{\mu}\right)^{N-2}(2 \pi)^{2} \sum_{m}^{\prime}\left(\frac{1}{m}\right)^{N-2} \alpha_{j}^{m} \alpha_{k}^{-m} .
$$

In this sum, higher Fourier modes $|m|>1$ are more and more suppressed as $N$ increases:

$$
\begin{aligned}
& \alpha_{j}^{+1} \cdot \alpha_{k}^{-1}+\alpha_{j}^{-1} \cdot \alpha_{k}^{+1}=-\lim _{N \text { even } \rightarrow \infty}(2 \pi)^{-2}\left(\frac{2 \pi}{\mathscr{P}_{d}}\right)^{N} \underbrace{\mathscr{Z}_{d \cdots d j k}^{(2)},}_{N}, \\
& \alpha_{j}^{+1} \cdot \alpha_{k}^{-1}-\alpha_{j}^{-1} \cdot \alpha_{k}^{+1}=-\lim _{N \text { odd } \rightarrow \infty}(2 \pi)^{-2}\left(\frac{2 \pi}{\mathscr{P}_{d}}\right)^{N} \underbrace{\mathscr{Z}_{N}^{(2)} \underbrace{}_{j k}}_{N} .
\end{aligned}
$$

Subtracting the contributions to $\mathscr{Z}_{d \ldots d j k}^{(2)}$ of the terms with $|m|=1$, we find

$$
\begin{aligned}
& \alpha_{j}^{+2} \cdot \alpha_{k}^{-2}+\alpha_{j}^{-2} \cdot \alpha_{k}^{+2} \\
& \quad=-\lim _{N \text { even } \rightarrow \infty} 2^{N}[(2 \pi)^{-2}\left(\frac{2 \pi}{\mathscr{P}_{d}}\right)^{N} \underbrace{\mathscr{Z}_{d \cdots d j k}^{(2)}}_{N}+\left(\alpha_{j}^{+1} \alpha_{k}^{-1}+\alpha_{j}^{-1} \alpha_{k}^{+1}\right)], \\
& \alpha_{j}^{+2} \cdot \alpha_{k}^{-2}-\alpha_{j}^{-2} \cdot \alpha_{k}^{+2} \\
& \quad=-\lim _{N \text { odd } \rightarrow \infty} 2^{N}[(2 \pi)^{-2}\left(\frac{2 \pi}{\mathscr{P}_{d}}\right)^{N} \underbrace{\mathscr{Z}_{d \cdots d j k}^{(2)}}_{N}+\left(\alpha_{j}^{+1} \alpha_{k}^{-1}-\alpha_{j}^{-1} \alpha_{k}^{+1}\right)],
\end{aligned}
$$


and so forth. In this way, step by step we obtain all products $\alpha_{j}^{m} \alpha_{k}^{-m}$, $j, k=1, \ldots,(d-1) ; m \neq 0$.

In a similar fashion, by taking appropriate limits of the invariant charges $\mathscr{Z}_{\mu_{1} \ldots \mu_{N}}^{(K)}$ with a fixed set of $n$ indices, which range individually over the integers between 1 and $(d-1)$, and $(N-n)$ indices equal to $d$, it is possible to extract the values of all products

$$
\alpha_{j_{1}}^{m_{1}} \ldots \alpha_{j_{n}}^{m_{n}} \text { for } m_{1}+\ldots+m_{n}=0, \quad j_{r} \in\{1, \ldots, d-1\}, \quad r=1, \ldots, n .
$$

In view of the constraint $\sum w_{\mu}^{\prime 2}(z)=0$ which corresponds to the following equations for the Fourier coefficients:

$$
\begin{gathered}
\sum_{j=1}^{d-1} \sum_{m=1}^{\infty} \alpha_{j}^{m} \cdot \alpha_{j}^{-m}=\frac{1}{2(2 \pi)^{2}} \sum_{\mu=1}^{d} \mathscr{P}_{\mu} \mathscr{P}_{\mu} \neq 0, \\
\sum_{j=1}^{d-1} \sum_{m=-\infty}^{+\infty} \alpha_{j}^{\ell+m} \cdot \alpha_{j}^{-m}=0 \text { for } \quad \ell \in \mathbb{Z} \backslash\{0\},
\end{gathered}
$$

neither all Fourier coefficients $\alpha_{j}^{m}$ with positive $m$ nor all Fourier coefficients $\alpha_{j}^{m}$ with negative $m$ vanish. Now, suppose for a minute that there exist indices $k$ and $\ell$ : $1 \leqq k, \ell \leqq(d-1)$ such that $\alpha_{k}^{1} \neq 0$ and $\alpha_{\ell}^{-1} \neq 0$. Then from the knowledge of the products

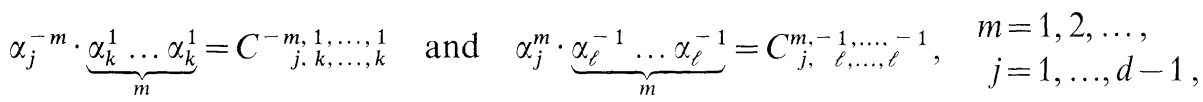

we can express $\alpha_{j}^{-m}$ and $\alpha_{j}^{+m}$ in terms of $\alpha_{k}^{1}$ and $\alpha_{\ell}^{-1}$, respectively,

$$
\begin{gathered}
\alpha_{j}^{-m}=C_{\substack{j, 1, \ldots, 1 \\
j, \ldots, k}}^{-m} /\left(\alpha_{k}^{1}\right)^{m}, \\
\alpha_{j}^{m}=C_{j,}^{m,-1, \ldots,-1}\left(\alpha_{\ell}, \ldots, \quad \ell /\left(\alpha_{\ell}^{-1}\right)^{m} .\right.
\end{gathered}
$$

Finally, when we replace $\alpha_{\ell}^{-1}$ by its expression in terms of $\alpha_{k}^{1}$ :

$$
\alpha_{\ell}^{-1}=C_{\ell, k}^{-1,1} / \alpha_{k}^{1} \quad \text { with } \quad C_{\ell, k}^{-1,1} \neq 0
$$

we arrive at $(m=1,2, \ldots)$

$$
\begin{aligned}
& \alpha_{j}^{m}=\left[\begin{array}{c}
C_{j}^{m},-1, \ldots,-1 \\
\ell, \ldots, \quad \ell
\end{array} /\left(C_{\ell, k}^{-1,1}\right)^{m}\right] \cdot\left(\alpha_{k}^{1}\right)^{m}, \quad j=1, \ldots, d-1 ; \quad \alpha_{d}^{m}=0, \\
& \alpha_{j}^{-m}=C_{j, k \ldots, k}^{-m, 1 \ldots, 1} \cdot\left(\alpha_{k}^{1}\right)^{-m}, \quad j=1, \ldots, d-1 ; \quad \alpha_{d}^{-m}=0, \\
& \alpha_{j}^{0}=\left(\mathscr{Z}_{j} / 2 \pi\right) \cdot\left(\alpha_{k}^{1}\right)^{0}, \quad j=1, \ldots, d-1, \\
& \alpha_{d}^{0}=\left(\mathscr{Z}_{d} / 2 \pi\right) \cdot\left(\alpha_{k}^{1}\right)^{0} .
\end{aligned}
$$

That is: All Fourier coefficients $\alpha_{j}^{m}$ of $d w_{\mu}(z(\zeta)) / d \zeta$ on the circle $|\zeta|=1$ are completely determined by the set of values of the invariant charges up to multiplication by $\lambda^{m}$, where $\lambda$ is a common non-vanishing complex number. This statement continues to hold also in the general case.

In general, when no indices $k$ and $\ell: 1 \leqq k, \ell \leqq(d-1)$ exist such that $\alpha_{k}^{1} \neq 0$ and $\alpha_{\ell}^{-1} \neq 0$, there are non-vanishing products

$$
\alpha_{j_{1}}^{m_{1}} \ldots \alpha_{j_{n}}^{m_{n}}, \quad j_{r} \in\{1, \ldots, d-1\}, \quad r=1, \ldots, \mathrm{n},
$$


such that $m=m_{1}+\ldots+m_{n}$ assumes its smallest positive value $m_{\min }$ :

$\alpha_{(k)}^{(p)}=\alpha_{k_{1}}^{p_{1}} \ldots \alpha_{k_{a}}^{p_{a}} \neq 0, \quad p_{1}+\ldots+p_{a}=m_{\min }>0, \quad k_{r} \in\{1, \ldots, d-1\}, \quad r=1, \ldots, a$, and such that $m=m_{1}+\ldots+m_{n}$ assumes its largest negative value $m_{\max }$ :

$\alpha_{(\ell)}^{(q)}=\alpha_{\ell_{1}}^{q_{1}} \ldots \alpha_{\ell_{b}}^{q_{b}} \neq 0, \quad q_{1}+\ldots+q_{b}=m_{\max }<0, \quad \ell_{s} \in\{1, \ldots, d-1\}, \quad s=1, \ldots, b$.

It turns out that

$$
-m_{\max }=m_{\min }
$$

because, if $\left|m_{\max }\right|>m_{\min }\left(\left|m_{\max }\right|<m_{\min }\right)$, the product

$$
\alpha_{(k)}^{(p)} \cdot \alpha_{(\ell)}^{(q)}=\alpha_{k_{1}}^{p_{1}} \ldots \alpha_{k_{a}}^{p_{a}{ }^{\prime}} \cdot \alpha_{\ell_{1}}^{q_{1}} \ldots \alpha_{\ell_{b}}^{q_{b}} \neq 0
$$

would furnish a counterexample against the maximality property of $m_{\max }$ (minimality property of $m_{\min }$ ).

For the case $m_{\min }=+1$, in order to arrive at the desired conclusion, all we have to do is to replace $\alpha_{k}^{1}$ and $\alpha_{\ell}^{-1}$ in the previous argument by $\alpha_{(k)}^{(p)}$ and $\alpha_{(\ell)}^{(q)}$, respectively.

For the case $m_{\min }>1$ we observe that for all non-vanishing products $\alpha_{j_{1}}^{m_{1}} \ldots \alpha_{j_{n}}^{m_{n}}$, $j_{r} \in\{1, \ldots, d-1\}, r=1, \ldots, n$, the sum $m=\left(m_{1}+\ldots+m_{n}\right)$ is an integer multiple of $m_{\min }$. In particular, this is true for all non-vanishing Fourier coefficients $\alpha_{j}^{m}$ with $m \neq 0: m=t \cdot m_{\min }$. Thus all non-vanishing Fourier coefficients can be expressed as follows:

$$
\begin{gathered}
\alpha_{j}^{m}=\left[C_{j,(\ell), \ldots,(\ell)}^{m,(q), \ldots,(q)} /\left(C_{(k)(\ell)}^{(p)(q)}\right)^{t}\right] \cdot\left(\alpha_{(k)}^{(p)}\right)^{t}, \quad j=1, \ldots, d-1 ; \quad t>0, \\
\alpha_{j}^{m}=C_{j,(k), \ldots,(k)}^{t,} \cdot \overbrace{(p),(p)}^{-t} \cdot\left(\alpha_{(k)}^{(p)}\right)^{t}, \quad j=1, \ldots, d-1 ; \quad t<0, \\
\alpha_{j}^{0}=\left(\mathscr{Z}_{j} / 2 \pi\right) \cdot\left(\alpha_{(k)}^{(p)}\right)^{0}, \quad j=1, \ldots, d-1, \\
\alpha_{d}^{m}=\delta_{m, 0} \cdot\left(\mathscr{Z}_{d} / 2 \pi\right) \cdot\left(\alpha_{(k)}^{(p)}\right)^{t} .
\end{gathered}
$$

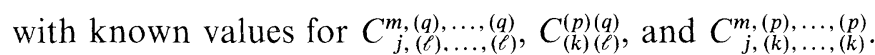

Finally, by setting $\lambda=\left(\alpha_{(k)}^{(p)}\right)^{1 / m_{\min }}$ (the branch of the root does not matter) again we arrive at the desired conclusion.

As explained before, the ambiguity of multiplication by powers of $\lambda$ corresponds to a rotation and scale reparametrization of the annulus $A$. In any case, the complete set of values of the invariant charges enables us to reconstruct up to global translations the region of the minimal surface which has the annulus $A$ as its parameter set and, moreover, by analytic continuation the corresponding complete minimal surface $\Sigma$.

\section{The Case When All Values of the Invariant Charges are Zero}

Let a complete minimal surface $\Sigma$ and an oriented smooth Jordan curve $\mathscr{C}$ on $\Sigma$ be given. Assume that all invariant charges vanish when evaluated along $\mathscr{C}$. In particular, the vanishing of the linear momenta $\mathscr{P}_{\mu}=\mathscr{Z}_{\mu} / i$ when evaluated along $\mathscr{C}$ implies that the smooth curve $\tilde{\mathscr{C}}$ conjugate to $\mathscr{C}$ [3] on the conjugate minimal surface $\tilde{\Sigma}$ is closed. Let the minimal surface $\Sigma$ in the neighbourhood of $\mathscr{C}$ be 
parametrized with the help of isothermal coordinates $z=x+i y$ from a two-fold connected domain $D$ which contains the unit circle

$$
x_{\mu}=\varphi_{\mu}(z), \quad z \in D,
$$

such that the unit circle of the complex $z$-plane $z=e^{i \theta}, 0 \leqq \theta \leqq 2 \pi$ is mapped (strictly monotonously) onto the curve $\mathscr{C}$. The complex Riemann surface $\Sigma+i \tilde{\Sigma}$ over $D$ is described by

$$
w_{\mu}=x_{\mu}+i y_{\mu}=\varphi_{\mu}(z)+i \psi_{\mu}(z)=w_{\mu}(z), \quad z \in D,
$$

where the functions $\psi_{\mu}(z)$ up to $d$ constants are defined by the Cauchy-Riemann equations,

$$
\begin{aligned}
& \partial_{x} \psi_{\mu}=-\partial_{y} \varphi_{\mu}, \quad x+i y \in D, \quad \mu=1, \ldots, d, \\
& \partial_{y} \psi_{\mu}=\partial_{x} \varphi_{\mu},
\end{aligned}
$$

as single-valued harmonic functions,

$$
w_{\mu}^{\prime}\left(e^{i \theta}\right) i e^{i \theta}=\frac{d}{d \theta} x_{\mu}(\ln |z|=0, \theta)+i p_{\mu}(\ln |z|=0, \theta) .
$$

Suppose that there exists a linear combination of the functions $w_{\mu}(z)$ :

$$
w(z) \doteq \sum_{\mu} c_{\mu} w_{\mu}(z), \quad c_{\mu}=\text { const } \in \mathbb{C},
$$

such that the unit circle $|z|=1$ and a sufficiently small neighbourhood of $|z|=1$ in $D$ are mapped one to one onto a Jordan curve $K$ in the complex $w$-plane and onto a twofold connected neighbourhood $P$ of $K$, respectively. Then the minimal surface $\Sigma$ is a continuation of a simply connected minimal surface spanned by $\mathscr{C}$, in other words: the curve $\mathscr{C}$ has trivial homology on $\Sigma$.

Proof. It is not difficult to show by induction on the rank of the tensors $W_{\mu_{1} \ldots \mu_{N}}^{|z|=1}(z)$ (we apologize for the slight abuse of notation) that vanishing values for all invariant charges $\mathscr{Z} \ldots$ imply the triviality of all the analytic functions $W_{\mu_{1} \ldots \mu_{N}}^{|z|=1}(z)$ :

$$
W_{\mu_{1} \ldots \mu_{N}}^{|z|=1}(z) \equiv 0, \quad z \in D
$$

Let $z=z(w), w \in P$, denote the inverse of the mapping $z \rightarrow w=w(z)$. Then

$$
w_{\mu}=x_{\mu}+i y_{\mu}=w_{\mu}(z(w))=\tilde{w}_{\mu}(w), \quad w \in P, \quad \mu=1, \ldots, d,
$$

is an isothermal parametrization of a neighbourhood of the Jordan curve " $\mathscr{C}+i \tilde{\mathscr{C}}$ " on $\Sigma+i \widetilde{\Sigma}$. We find also in this parametrization that (abusing the notation once more)

$$
W_{\mu_{1} \ldots \mu_{N}}^{K}(w) \equiv 0, \quad \mu_{i} \in\{1, \ldots, d\} ; \quad i=1, \ldots, N ; \quad N=1,2, \ldots .
$$

In particular, this implies

$$
0=\oint_{K} d w w^{n} \tilde{w}_{\mu}(w), \quad \mu=1, \ldots, d ; \quad n=0,1, \ldots
$$

The single-valued analytic functions $\tilde{w}_{\mu}(w), w \in P$, permit a Cauchy integral representation

$$
-\tilde{w}_{\mu}(w)=\frac{1}{2 \pi i}\left(\oint_{K_{1}}-\oint_{K_{2}}\right) d \hat{w} \frac{\tilde{w}_{\mu}(\hat{w})}{\hat{w}-w}=\tilde{w}_{\mu}^{(1)}(w)-\tilde{w}_{\mu}^{(2)}(w)
$$


with

$$
\tilde{w}_{\mu}^{(i)}(w)=\frac{1}{2 \pi i} \oint_{K_{\iota}} d \hat{w} \frac{\tilde{w}_{\mu}(\hat{w})}{\hat{w}-w}, \quad i=1,2 .
$$

Here $K_{1}$ and $K_{2}$ form the boundaries of the parameter region $P, K_{1}\left(K_{2}\right)$ lying in the interior (exterior) of the curve $K$.

By their defining integral representation, the functions $\tilde{w}_{\mu}^{(2)}(w)$ can be analytically continued into the interior of $K$. Hence, trivially

$$
\oint_{K} d w w^{n} \tilde{w}_{\mu}^{(2)}(w)=0, \quad \mu=1, \ldots, d ; \quad n=0,1, \ldots,
$$

so that the fact that all values of the invariant charges are zero implies

$$
\oint_{K} d w w^{n} \tilde{w}_{\mu}^{(1)}(w)=0, \quad \mu=1, \ldots, d ; \quad n=0,1, \ldots
$$

Again, by the defining integral representation, the functions $\tilde{w}_{\mu}^{(1)}(w)$ can be analytically continued into the exterior of $K$. In particular, they can be analytically continued to the outside $\left|w-w_{0}\right| \geqq R_{0}$ of the largest of the circles of convergence for the Laurent expansions,

$$
\tilde{w}_{\mu}^{(1)}(w)=\sum_{\ell=-\infty}^{-1} a_{\mu}^{\ell}\left(w-w_{0}\right)^{\ell}, \quad \mu=1, \ldots, d,
$$

around a conveniently chosen point $w_{0}$ in the interior of $K_{1}$. Hence

$$
\begin{gathered}
\frac{1}{2 \pi i} \oint_{K} d w w^{n} \tilde{w}_{\mu}^{(1)}(w)=\frac{1}{2 \pi i} \oint_{\left|w-w_{0}\right|=R} d w w^{n} \tilde{w}_{\mu}^{(1)}(w)=\sum_{k=0}^{n}\left(\begin{array}{l}
n \\
k
\end{array}\right) w_{0}^{n-k} a_{\mu}^{-k-1}, \\
\mu=1, \ldots, d ; \quad n=0,1, \ldots ; \quad R>R_{0} .
\end{gathered}
$$

The vanishing of the integrals on the extreme left-hand side of the chain of equations implies

$$
a_{\mu}^{-n-1}=0, \quad \mu=1, \ldots, d ; \quad n=0,1, \ldots
$$

Thus

$$
\tilde{w}_{\mu}^{(1)}(w) \equiv 0, \quad \mu=1, \ldots, d,
$$

or equivalently,

$$
\tilde{w}_{\mu}(w) \equiv \tilde{w}_{\mu}^{(2)}(w)
$$

and $\tilde{w}_{\mu}(w)$ can be analytically continued into the interior of $K$. That is, the part of the complete minimal surface $\Sigma$ bounded by the curve $\mathscr{C}$ and corresponding to points in the interior of $K$ is simply connected, and it coincides with a minimal surface spanned by the curve $\mathscr{C}$.

\section{Conclusions}

The preceding analysis shows that a given algebraic basis of the invariant charges of the Nambu-Goto string theory [2] provides a complete description of the minimal surfaces (of class $\mathscr{I}$ ) in terms of conformally invariant parameters. In this sense the invariant charges are natural candidates for physically relevant operators in the quantum theory of strings. This view is corroborated by the relative simplicity of the WKB-renormalization of these charges [3] and the fact 
that one and - as suggested by Sect. IV above - only one string state, the ground state $\psi(\mathscr{C})$ of [8], is invariant in WKB-approximation under the symmetry transformations generated by the invariant charges.

If one compares the structure of the invariant charges in the original Minkowski version of the Nambu-Goto theory with the structure of the higher conserved local and non-local charges in the two-dimensional $\mathrm{O}(\mathrm{N})$ non-linear $\sigma$-model [9], one might wonder whether it is possible to replace equivalently the information contained in the values of (an algebraic basis of) the invariant charges for some "intermediate" generic homology (initial data) of a trajectory surface $\Sigma$ by the sum of simpler information corresponding to more specific fixed subsets of the invariant charges evaluated for appropriately many time-like asymptotic homologies of $\Sigma$. This is an open problem.

Acknowledgements. One of the present authors (K.P.) would like to thank the staff of the MaxPlanck-Institut für Physik und Astrophysik for the kind hospitality extended to him during his stay in Munich in the spring of 1986 when part of this work was done. Financial support by the Max-Planck-Gesellschaft is gratefully acknowledged.

\section{References}

1. Pohlmeyer, K.: A group-theoretical approach to the quantization of the free relativistic closed string. Phys. Lett. 119 B, 100 (1982)

2. Pohlmeyer, K., Rehren, K.-H.: Algebraic properties of the invariant charges of the NambuGoto theory. Commun. Math. Phys. 105, 593 (1986)

3. Pohlmeyer, K.: The invariant charges of the Nambu-Goto theory in WKB-approximation: renormalization. Commun. Math. Phys. 105, 629 (1986)

4. Pohlmeyer, K., Rehren, K.-H.: The algebra formed by the invariant charges of the NambuGoto theory: identification of a maximal abelian subalgebra. Commun. Math. Phys. 114, 55-68 (1988)

5. Nitsche, J.C.C.: Vorlesungen über Minimalflächen; Chap. VI, Sect. 3.3. Die Grundlehren der mathematischen Wissenschaften, Band 199. Berlin, Heidelberg, New York: Springer 1975

6. Ahlfors, L.V.: Complex analysis (an introduction to the theory of analytic functions of one complex variable), $3^{\text {rd }}$ edn., Chap. 6. New York: McGraw-Hill 1979

7. Throughout the subsequent analysis we shall assume that the complete minimal surfaces $\Sigma$ are orientable. Should they fail to be so, we replace the canonical region $\Omega$ by a two-fold covering thereof as explained in Weil, H.: Die Idee der Riemannschen Fläche, $3^{\text {rd }}$ edn., p. 48. Stuttgart: Teubner 1955

8. Lüscher, M., Symanzik, K., Weisz, P.: Anomalies of the free loop wave equation in the WKBapproximation. Nucl. Phys. B 173, 365 (1980)

9. Polyakov, A.M.: Hidden symmetry of the two-dimensional chiral fields. Phys. Lett. 72 B, 224 (1977/78)

Lüscher, M., Pohlmeyer, K.: Scattering of massless lumps and non-local charges in the twodimensional classical non-linear $\sigma$-model. Nucl. Phys. B 137, 46 (1978)

Lüscher, M.: Quantum non-local charges and absence of particle production in the twodimensional non-linear $\sigma$-model. Nucl. Phys. B 135, 1 (1978)

Communicated by K. Osterwalder

Received April 8, 1987 